\title{
Field Theoretic Models of the Dispersive Dielectric Medium
}

\author{
Ż. Artyszuk and A. Bechler \\ Institute of Physics, University of Szczecin \\ Wielkopolska 15, 70-451 Szczecin, Poland
}

\begin{abstract}
The purpose of this paper is a description of the dispersive dielectric medium, both linear and nonlinear, from first principles using the field theoretic methods based on the Feynman path integrals over classical trajectories. The main idea is to use notion of effective fields, in the present case the electromagnetic field modified by presence of a polarizable medium. Interaction of the field with the medium on the microscopic level is described by a modified Hopfield Lagrangian containing terms corresponding to the electromagnetic field, the matter polarization field modelled by harmonic oscillators with some resonance frequency and other matter fields describing the degrees of freedom responsible for absorption in the medium (reservoir fields). The polarization field is coupled both to the electric field and the reservoir fields. Effective theory is obtained by elimination of the matter degrees of freedom which is achieved by functional integration over all matter fields. For a linear medium all calculations can be done exactly leading to the effective Lagrangian from which, among others, an expression for frequency dependent dielectric constant can be extracted. Explicit form of the dielectric constant depends on the way by which the polarization field couples to the reservoir fields. In particular, uniform coupling to all reservoir modes gives the standard Lorentz oscillator model, and for any type of coupling the Lorentz form of dielectric constant is retrieved for frequencies close to the resonance. For weak damping the dispersion $\omega(k)$ is little sensitive to the form of coupling leading to polariton modes not different from those of the Lorentz model. It is also outlined briefly how the functional integration method could be used to description of nonlinear effects in the medium.
\end{abstract}

PACS numbers: 42.65.-k, 12.20.--m

\section{Introduction}

The purpose of this paper is to present a model of the dispersive dielectric medium, both linear and nonlinear, based on field theoretical method of integration 
over classical histories of a physical system, i.e. Feynman path integrals $[1,2]$. The main idea behind application of this method to the description of polarizable media is that on the macroscopic level electromagnetic field in a dielectric medium is an example of an effective field, where the external electric field is modified by polarization of the medium. It is therefore an important question how this bulk material effects can be accounted for taking microscopic Lagrangian of the medium and electromagnetic field as starting point. There is a wide body of literature dealing with this issue; the approach presented here is based on the Hopfield model [3], which has been extended to include damping by Huttner and Barnett [4]. In this last paper the full quantum theory was developed based on the method of canonical quantization. The main goal is to eliminate matter degrees of freedom corresponding to the polarization of the medium and to damping modes described by a set of harmonic oscillators coupled to the polarization field. This program has been performed with the use of Feynman path integrals in [5] including full quantization of the electromagnetic field in a dispersive dielectric. In this note we concentrate our attention on the properties of the frequency dependent dielectric constant, which does not require quantization of the electromagnetic field [6]. Main novel features of present publication are contained in the discussion of various models of the dielectric constant following from different couplings to the damping modes also beyond the standard Lorentz oscillator model, and also in an outline of how the functional integration methods can be used to describe the nonlinear effects in an approximate way.

The paper is organized as follows. In Sec. 2 we give general outline of the application of path integral method to the dispersive dielectric - details can be found in [5]. Section 3 contains discussion of the Lorentz oscillator model in the context of the present approach. It can be viewed as an extension and modification of the results obtained in [7]. In particular, we show that this model corresponds to uniform coupling of the atomic oscillations to the "reservoir" fields responsible for damping. We also show that any type of the coupling to the reservoir fields leads to the Lorentz expression for the dielectric function in the vicinity of resonance frequency. This section contains also discussion of a "nonconventional" coupling which cannot be directly related to the Lorentz model in the entire frequency range. The main result here is that, despite of these differences, the structure of the dispersion $\omega(k)$ is for realistic values of the parameters nearly identical to that obtained from Lorentz model. Section 4 is devoted to a short description of how nonlinear effects can be described and managed using the path integral method, and Sec. 5 contains conclusions.

\section{General outline of the method}

The microscopic action of a dielectric medium interacting with the electromagnetic field is assumed to have the form $[4,5]$ : 
$S\left[\boldsymbol{E}, \boldsymbol{B}, \boldsymbol{P}, \boldsymbol{Y}_{\omega}\right]$

$$
\begin{aligned}
& =\int \mathrm{d} t \int \mathrm{d}^{3} x\left[\frac{\varepsilon_{0}}{2} \boldsymbol{E}^{2}-\frac{1}{2 \mu_{0}} \boldsymbol{B}^{2}+\frac{1}{2 \varepsilon_{0} \omega_{0}^{2} \beta}\left(\dot{\boldsymbol{P}}^{2}-\omega_{0}^{2} \boldsymbol{P}^{2}\right)-\boldsymbol{E} \cdot \boldsymbol{P}\right] \\
& +\int \mathrm{d} t \int \mathrm{d}^{3} x \int_{0}^{\infty} \mathrm{d} \omega^{\prime}\left[\frac{\rho}{2} \dot{\boldsymbol{Y}}_{\omega^{\prime}}^{2}-\frac{\rho \omega^{\prime 2}}{2} \boldsymbol{Y}_{\omega^{\prime}}^{2}-f\left(\omega^{\prime}\right) \boldsymbol{P} \cdot \boldsymbol{Y}_{\omega^{\prime}}\right] \\
& +\int \mathrm{d} t \int \mathrm{d}^{3} x F(\boldsymbol{P}(t, x)),
\end{aligned}
$$

where $\boldsymbol{P}$ is the matter polarization field modelled by a collection of harmonic oscillators with the eigenfrequency $\omega_{0}$. The coefficient $\beta$ has the meaning of dimensionless static polarizability. The coupling between the electric field and dipole moment of the medium is of the standard type $\boldsymbol{E} \cdot \boldsymbol{P}$. The oscillator fields $\boldsymbol{Y}_{\omega}$ mimic degrees of freedom corresponding to the energy absorbing states and coupling of the polarization field with these "reservoir" fields are responsible for the absorption in the medium and, in consequence, to the appearance of the imaginary part of dielectric constant. The function $f\left(\omega^{\prime}\right)$ describes the way in which the polarization oscillators are coupled to the reservoir oscillators of various frequencies $\omega^{\prime}$. Nonlinear effects are described by the term $\int \mathrm{d} t \int \mathrm{d}^{3} x F(\boldsymbol{P}(t, \boldsymbol{x}))$ [8], where the function $F(\boldsymbol{P})$ may in principle have an arbitrary form. For the case of a linear medium, considered in Secs. 2 and 3, this function is put equal to zero. All fields in (2.1) depend on time and coordinates but no spatial derivatives appear in the Lagrangian density, so that the dielectric constant does not depend on the wave vector but only on frequency. This corresponds to temporal nonlocality of the effective action and spatial locality, i.e. no spatial dispersion.

To find the effective action of the (classical) electromagnetic field in a dispersive dielectric medium we eliminate matter degrees of freedom by the path integral method. This leads to the ground state persistence amplitude in the form [5]:

$$
C[\boldsymbol{E}, \boldsymbol{B}]=\exp \left(\frac{\mathrm{i}}{\hbar} S_{\mathrm{eff}}[\boldsymbol{E}, \boldsymbol{B}]\right)=\int[\mathrm{d} \boldsymbol{P}]\left[\mathrm{d} \boldsymbol{Y}_{\omega}\right] \exp \left(\frac{\mathrm{i}}{\hbar} S\left[\boldsymbol{E}, \boldsymbol{B}, \boldsymbol{P}, \boldsymbol{Y}_{\omega}\right]\right),
$$

where the effective action is denoted by $S_{\text {eff. }}$. For a linear medium all functional integrals are Gaussian and can be performed explicitly. As details of this calculation have been presented elsewhere [5], we give here only final results. Classical effective action has the form

$$
\begin{aligned}
& S_{\mathrm{eff}}[\boldsymbol{E}, \boldsymbol{B}]=\int \mathrm{d} t \int \mathrm{d}^{3} x\left(\frac{\varepsilon_{0}}{2} \boldsymbol{E}^{2}-\frac{1}{2 \mu_{0}} \boldsymbol{B}^{2}\right) \\
& \quad+\frac{1}{2} \int \mathrm{d} t \int \mathrm{d} t^{\prime} \int \mathrm{d}^{3} x \boldsymbol{E}(t, \boldsymbol{x}) \Gamma\left(t-t^{\prime}\right) \boldsymbol{E}\left(t^{\prime}, \boldsymbol{x}\right),
\end{aligned}
$$

where Fourier transform of the propagator $\Gamma\left(t-t^{\prime}\right)$ is given by [5]:

$$
\widetilde{\Gamma}(\omega)=\frac{\varepsilon_{0} \omega_{0}^{2} \beta}{\omega_{0}^{2}-\omega^{2}-\omega^{2} \lambda_{\mathrm{F}}(\omega)},
$$


with

$$
\lambda_{\mathrm{F}}(\omega)=\frac{1}{\rho^{2}} \int_{0}^{\infty} \mathrm{d} \omega^{\prime} \frac{\left|v\left(\omega^{\prime}\right)\right|^{2}}{\omega^{\prime 2}-\omega^{2}=\mathrm{i} \epsilon}
$$

and the scaled coupling function $v(\omega)$ is given by $v(\omega)=\left(\varepsilon_{0} \omega_{0}^{2} \beta \rho\right)^{1 / 2} f(\omega)$.

It can be clearly seen from (2.3) that the effective action is nonlocal in time, with the nonlocality described by the propagator $\Gamma\left(t-t^{\prime}\right)$. In a local theory of electromagnetic field one can define the displacement field $\boldsymbol{D}(t, \boldsymbol{x})$ by [9]:

$$
\boldsymbol{D}(t, \boldsymbol{x})=\frac{\partial \mathcal{L}}{\partial \boldsymbol{E}(t, \boldsymbol{x})},
$$

where $\mathcal{L}$ is a local Lagrangian density of the electromagnetic field. Natural generalization of (2.6) to the case of nonlocal theories is to write (2.3) using time Fourier components of the fields, which gives for the Lagrangian density in the $(\omega, x)$ space $[5]$ :

$$
\mathcal{L}_{\text {eff }}(\boldsymbol{E}, \boldsymbol{B})=\varepsilon_{0}|\boldsymbol{E}(\omega, \boldsymbol{x})|^{2}-\frac{1}{\mu_{0}}|\boldsymbol{B}(\omega, \boldsymbol{x})|^{2}+\widetilde{\Gamma}(\omega)|\boldsymbol{E}(\omega, \boldsymbol{x})|^{2},
$$

and determine the displacement field as

$$
\boldsymbol{D}(\omega, \boldsymbol{x})=\frac{\partial \mathcal{L}_{\mathrm{eff}}}{\partial \boldsymbol{E}^{*}(\omega, \boldsymbol{x})},
$$

which immediately leads to

$$
\boldsymbol{D}(\omega, \boldsymbol{x})=\varepsilon_{0} \boldsymbol{E}(\omega, \boldsymbol{x})+\widetilde{\Gamma}(\omega) \boldsymbol{E}(\omega, \boldsymbol{x}) .
$$

To avoid "double counting" [4,5] one can use formulae (2.7), (2.8) and (2.9) only for $\omega>0$. The dielectric constant for real positive frequencies is therefore given by

$$
\varepsilon_{+}(\omega)=1+\frac{1}{\varepsilon_{0}} \widetilde{\Gamma}(\omega)
$$

Analytic continuation to negative frequencies can be done according to $\varepsilon_{-}(\omega)=$ $\varepsilon_{+}^{*}(-\omega)$, which in the final result gives [5]:

$$
\varepsilon(\omega)=1+\frac{\omega_{0}^{2} \beta}{\omega_{0}^{2}-\omega^{2}-\omega^{2} \lambda_{\mathrm{R}}(\omega)},
$$

where

$$
\lambda_{\mathrm{R}}(\omega)=\frac{1}{\rho^{2}} \int_{0}^{\infty} \mathrm{d} \omega^{\prime} \frac{\left|v\left(\omega^{\prime}\right)\right|^{2}}{\omega^{2}-\omega^{2}-\mathrm{i} \epsilon \operatorname{sgn}(\omega)} .
$$

Equation (2.12) gives the basic expression for the function $\lambda_{R}(\omega)$, which determines properties of the dielectric constant as function of frequency. Assuming that $|v(\omega)|^{2}$ is an even square integrable function [4] one can write (2.12) in the form [5]:

$$
\lambda_{\mathrm{R}}(\omega)=\frac{1}{2 \rho^{2} \omega} \int_{-\infty}^{\infty} \mathrm{d} \omega^{\prime} \frac{\left|v\left(\omega^{\prime}\right)\right|^{2}}{\omega^{\prime}-\omega-\mathrm{i} \epsilon} .
$$

Expression (2.11) for the dielectric constant will be discussed in the next section, mainly from the point of view of its dependence on the shape of the coupling function $v(\omega)$ and comparison with existing models. This last issue will be considered also in connection with the recent publication by Wubs and Suttorp [7]. 


\section{Models of the dielectric constant in a linear dispersive medium}

We shall consider now properties of the dielectric constant following from (2.11) and (2.12) with various shapes of the reservoir coupling function $v(\omega)$. First of all we compare the present approach with the standard Lorentz oscillator model of the dielectric medium, in which [7]:

$$
\varepsilon_{\mathrm{L}}(\omega)=1+\frac{\omega_{\mathrm{c}}^{2}}{\omega_{0}^{2}-\omega^{2}-2 \mathrm{i} \omega \gamma} .
$$

To find what type of coupling to the reservoir fields corresponds to the Lorentz oscillator model we have to identify imaginary part of $\lambda_{\mathrm{R}}(\omega)$, which according to $(2.12)$ is

$$
\operatorname{Im} \lambda_{\mathbf{R}}(\omega)=\frac{\pi}{2 \rho^{2} \omega}|v(\omega)|^{2} .
$$

Comparing (2.11) with (3.1) we see that $\omega^{2} \operatorname{Im} \lambda_{R}(\omega)=2 \omega \gamma$ which immediately gives

$$
|v(\omega)|^{2}=\frac{4 \gamma \rho^{2}}{\pi} .
$$

This shows that Lorentz oscillator model corresponds to uniform coupling of the polarization field to the reservoir oscillators or frequency independent damping. Let us note however that using formula (2.13) leads to a divergent integral and one should introduce then a cut-off assuming the coupling function e.g. in the form [7]:

$$
|v(\omega)|^{2}=\frac{4 \gamma \rho^{2}}{\pi} \frac{b^{2}}{b^{2}+\omega^{2}},
$$

which has the limit equal to (3.3) when the cut-off parameter $b$ tends to infinity. Substitution of (3.4) to (2.13) gives a convergent integral. The above derivation shows that the Lorentz oscillator model is a special case of the general form of the dielectric constant (2.11) and (2.12), contrary to what has been argued in [6], that the Lorentz oscillator model is not compatible with damped Hopfield model analyzed in the scheme of canonical quantization in [4]. Also, in contrast with the derivation of this model presented in [7] in a similar scheme, it is by no means necessary to introduce a cut-off to get convergent integral for $\lambda_{\mathrm{R}}(\omega)$. On the other hand, use of the cut-off leads to an interaction of the polarization field with damping modes of the medium for which coupling to high frequency reservoir oscillators is suppressed.

The Lorentz oscillator model corresponds to a situation in which the damping constant $\gamma$ is frequency independent. On the other hand, dielectric constant given by Eq. (2.11) characterizes a medium for which the damping constant would in general depend on frequency; we shall show now that, independent of the shape of the coupling function $v(\omega)$, the dielectric function reduces to the Lorentz oscillator form in the vicinity of resonance frequency determined by 


$$
\omega_{\text {res }}^{2}-\omega_{0}^{2}+\omega_{\text {res }}^{2} \operatorname{Re} \lambda_{\mathrm{R}}\left(\omega_{\text {res }}\right)=0
$$

The denominator of (2.11) can be written as $f\left(\omega^{2}\right)+i \omega^{2} \operatorname{Im} \lambda_{\mathrm{R}}(\omega)$, where $f\left(\omega^{2}\right)=$ $\omega^{2}-\omega_{0}^{2}+\omega^{2} \operatorname{Re} \lambda_{\mathbf{R}}(\omega)$ - let us note that real part of $\lambda_{\mathbf{R}}$ is a function of $\omega^{2}$ [cf. (2.12)]. Close to the resonance frequency we have $f\left(\omega^{2}\right) \approx f^{\prime}\left(\omega_{\text {res }}^{2}\right)\left(\omega^{2}-\omega_{\text {res }}^{2}\right)$. Using (3.4) and approximating slowly varying function $v(\omega)$ by its value at resonance frequency, we can write (2.11) approximately as

$$
\varepsilon(\omega) \approx 1+\frac{\omega_{\mathrm{C}}^{2}}{\omega_{\mathrm{res}}^{2}-\omega^{2}-2 \mathrm{i} \omega \gamma},
$$

with $\omega_{\mathrm{c}}^{2}=\omega_{0}^{2} \beta / f^{\prime}\left(\omega_{\text {res }}^{2}\right)$ and $\gamma=\left(\pi / 4 \rho^{2}\right)\left|v\left(\omega_{\text {res }}\right)\right|^{2} / f^{\prime}\left(\omega_{\text {res }}^{2}\right)$.

The dispersion, i.e. dependence of the frequency on the real wave vector $k$ is determined by equation

$$
\omega^{2} \varepsilon(\omega)-c^{2} k^{2}=0 .
$$

For complex dielectric constant this leads to complex polariton modes with complex frequency having negative imaginary part. In the Lorentz oscillator model there are two polariton branches with a band gap between them [3].

The Lorentz oscillator model has been analyzed in the framework of an approach similar to the present one previously [7,6], also with the use of the cut-off function of the type (3.4) [7]. We shall consider now a "nonconventional" coupling function which cannot be directly related to this model and has the form

$$
v(\omega)=a \rho \omega_{0}^{3 / 2} \frac{\omega}{\omega^{2}+b^{2}} .
$$

There are two phenomenological parameters characterizing this coupling, $a$ and $b$. The first one is related to strength of the coupling, the second determines width of the coupling function. With increasing $b$ more high frequency modes of the reservoir are coupled to the polarization oscillators, and maximum value of $v(\omega)$ decreases leading to weaker coupling to the reservoir fields. Let us note also that with $v(\omega)$ given by (3.8) there is no coupling to zero frequency modes, contrary to (3.4). The coupling (3.8) fulfills also the requirements for $v(\omega)$ mentioned e.g. in [4], i.e. it is square integrable and does not vanish for nonzero frequencies. The function $\lambda_{\mathrm{R}}(\omega)(2.12)$ is

$$
\lambda_{\mathrm{R}}(\omega)=-\frac{a^{2} \pi}{4 b_{0}} \frac{1}{\left(\omega / \omega_{0}+\mathrm{i} b_{0}\right)^{2}},
$$

where $b_{0}=b / \omega_{0}$. The resonance frequency [cf. (3.5)] does not differ much from $\omega_{0}$. A reasonable choice for the parameter $b$ is e.g. $b=10 \omega_{0}$, which couples the polarization field to a relatively broad region of the reservoir oscillators. In the vicinity of the resonance frequency dielectric constant is given by (3.6), and assuming that $\gamma \approx 10^{8} \mathrm{~s}^{-1}$ we find that for $\omega_{0}$ in optical region the parameter $a$ may be chosen as $a=0.1$. The real and imaginary parts of the dielectric constant in narrow region around $\omega_{0}$ are shown in Fig. 1. The shape of $\varepsilon(\omega)$ has a less or more standard form with a detailed structure visible only in a very narrow region close to the resonance 

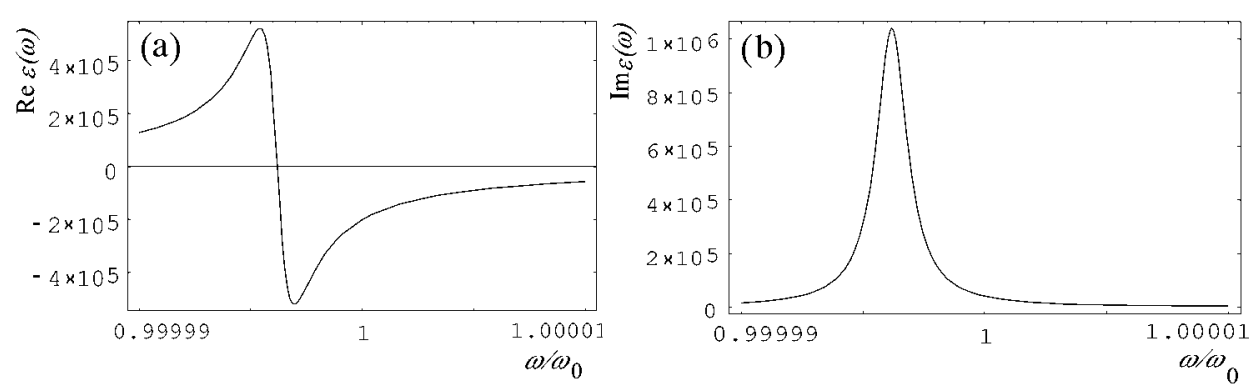

Fig. 1. Dielectric constant for the coupling function (3.8) in the vicinity of the resonance frequency.
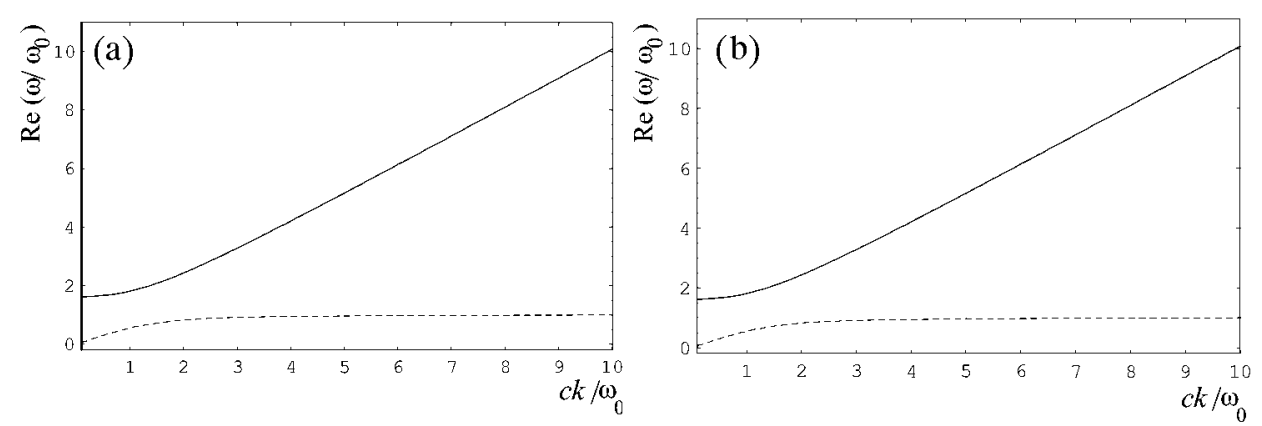

Fig. 2. Real part of the dispersion relation (3.7) for the coupling function (3.8) (a), and the Lorentz oscillator model (3.1) (b). Values of the parameters $a, b$ and of the damping constant $\gamma$ are given in the text. For these values of the parameters imaginary parts of $\omega(k)$ vanish for both branches.

frequency, which is slightly smaller than $\omega_{0}$. Solutions of the dispersion relation (3.7) are shown in Fig. 2a. There are two polariton branches, with the values of the parameters mentioned above practically identical to the solutions of (3.7) for the Lorentz oscillator model, shown for comparison in Fig. 2b. The band gap, i.e. region of frequencies for which (3.7) has no solution for any real $k$, corresponds approximately to $\omega_{0} \leq \omega \leq 1.6 \omega_{0}$. For frequencies in this region real part of the refraction coefficient $n(\omega)=\sqrt{\varepsilon(\omega)}$ vanishes and its imaginary part may acquire large values. Using the coupling function (3.8) one obtains also a third purely imaginary mode with $\operatorname{Im} \varepsilon \approx-10$. A similar purely imaginary "cut-off mode" [7] also appears after using the coupling function (3.4); this mode disappears when $b \rightarrow \infty$ and is therefore not present in the Lorentz oscillator model.

\section{Nonlinear effects in a dispersive dielectric medium}

To account for nonlinear effects we use the microscopic action (2.1) with nonvanishing $F(\boldsymbol{P})$. For instance in the case of a Kerr-type dielectric we would have 
$F(\boldsymbol{P})=\frac{1}{4} \alpha(\boldsymbol{P} \cdot \boldsymbol{P})^{2}[8]$. In general the functional integrations are not Gaussian anymore and can be evaluated only with the use of some type of perturbative methods. One of the possibilities is to use effective action after eliminating degrees of freedom of the reservoir field

$$
\begin{aligned}
& S_{\text {eff }}^{(1)}[\boldsymbol{E}, \boldsymbol{B} ; \boldsymbol{P}]=S_{\mathrm{em}}[\boldsymbol{E}, \boldsymbol{B}] \\
& \quad+\frac{1}{2} \int \mathrm{d} t \int \mathrm{d}^{3} x \frac{1}{\varepsilon_{0} \omega_{0}^{2} \beta}\left(\dot{\boldsymbol{P}}^{2}-\omega_{0}^{2} \boldsymbol{P}^{2}\right)-\int \mathrm{d} t \int \mathrm{d}^{3} x \boldsymbol{E} \cdot \boldsymbol{P} \\
& \quad+\frac{1}{2} \int \mathrm{d} t \int \mathrm{d} t^{\prime} \int \mathrm{d}^{3} x \int_{0}^{\infty} \mathrm{d} \omega^{\prime} f\left(\omega^{\prime}\right)^{2} \frac{1}{\rho} \dot{\boldsymbol{P}}(t) D_{\mathrm{F}}\left(t-t^{\prime} ; \omega^{\prime}\right) \dot{\boldsymbol{P}}\left(t^{\prime}\right) \\
& +\int \mathrm{d} t \int \mathrm{d}^{3} x F(\boldsymbol{P}(t, x)),
\end{aligned}
$$

where $[5]$ :

$$
D_{\mathrm{F}}\left(t-t^{\prime} ; \omega^{\prime}\right)=\int_{-\infty}^{\infty} \frac{\mathrm{d} \omega}{2 \pi} \frac{\exp \left[-\mathrm{i} \omega\left(t-t^{\prime}\right)\right]}{\omega^{\prime 2}-\omega^{2}-\mathrm{i} \epsilon} .
$$

Treating the polarization field as the dynamical variable we obtain from least action principle the following equation for $\boldsymbol{P}$ :

$$
\begin{aligned}
\frac{\partial^{2} \boldsymbol{P}}{\partial t^{2}} & +\omega_{0}^{2} \boldsymbol{P}+\varepsilon_{0} \frac{\beta \omega_{0}^{2}}{\rho} \int \mathrm{d} t^{\prime} \int_{0}^{\infty} \mathrm{d} \omega^{\prime} f\left(\omega^{\prime}\right)^{2} \frac{\partial^{2}}{\partial t^{2}} D_{\mathrm{F}}\left(t-t^{\prime} ; \omega^{\prime}\right) \boldsymbol{P}\left(t^{\prime}\right) \\
& =-\varepsilon_{0} \beta \omega_{0}^{2} \boldsymbol{E}+\varepsilon_{0} \beta \omega_{0}^{2} \frac{\partial F}{\partial \boldsymbol{P}}
\end{aligned}
$$

or

$$
\begin{aligned}
\frac{\partial^{2} \boldsymbol{P}}{\partial t^{2}} & +\omega_{0}^{2} \boldsymbol{P}+\varepsilon_{0} \frac{\beta \omega_{0}^{2}}{\rho} \int \mathrm{d} t^{\prime} F\left(t-t^{\prime}\right) \boldsymbol{P}\left(t^{\prime}\right) \\
& =-\varepsilon_{0} \beta \omega_{0}^{2} \boldsymbol{E}+\varepsilon_{0} \beta \omega_{0}^{2} \frac{\partial F}{\partial \boldsymbol{P}}
\end{aligned}
$$

where

$$
F\left(t-t^{\prime}\right)=\int_{0}^{\infty} \mathrm{d} \omega^{\prime} f\left(\omega^{\prime}\right)^{2} \frac{\partial^{2}}{\partial t^{2}} D_{\mathrm{F}}\left(t-t^{\prime} ; \omega^{\prime}\right) .
$$

Green function $\Gamma\left(t-t^{\prime}\right)$ of Eq. (4.4) fulfills 


$$
\begin{aligned}
& \left(\frac{\partial^{2}}{\partial t^{2}}+\omega_{0}^{2}\right) \Gamma\left(t-t^{\prime}\right)+\varepsilon_{0} \frac{\beta \omega_{0}^{2}}{\rho} \int \mathrm{d} t^{\prime \prime} F\left(t-t^{\prime \prime}\right) \Gamma\left(t^{\prime \prime}-t^{\prime}\right) \\
& =\varepsilon_{0} \beta \omega_{0}^{2} \delta\left(t-t^{\prime}\right),
\end{aligned}
$$

and it can be easily shown that its Fourier transform is given by (2.4) and (2.5). If a solution of the homogeneous equation is not necessary (e.g. when the electric field vanishes in the "remote past" and is switched on gradually), the solution of Eq. (4.4) can be formally written as

$$
\boldsymbol{P}(t)=-\int \mathrm{d} t^{\prime} \Gamma\left(t-t^{\prime}\right) \boldsymbol{E}\left(t^{\prime}\right)+\int \mathrm{d} t^{\prime} \Gamma\left(t-t^{\prime}\right) \frac{\partial F}{\partial \boldsymbol{P}\left(t^{\prime}\right)} .
$$

If, for example $F(\boldsymbol{P})=\frac{1}{4} \alpha(\boldsymbol{P} \cdot \boldsymbol{P})^{2}$, then $\partial F / \partial \boldsymbol{P}=\alpha(\boldsymbol{P} \cdot \boldsymbol{P}) \boldsymbol{P}$ and the integral equation for the polarization field has the form

$$
\boldsymbol{P}\left(t^{\prime}\right)=-\int \mathrm{d} t^{\prime} \Gamma\left(t-t^{\prime}\right) \boldsymbol{E}\left(t^{\prime}\right)+\alpha \int d t^{\prime} \Gamma\left(t-t^{\prime}\right)\left[\boldsymbol{P}\left(t^{\prime}\right) \cdot \boldsymbol{P}\left(t^{\prime}\right)\right] \boldsymbol{P}\left(t^{\prime}\right)
$$

The first-order polarization is given by

$$
\boldsymbol{P}^{(1)}(t)=-\int \mathrm{d} t^{\prime} \Gamma\left(t-t^{\prime}\right) \boldsymbol{E}\left(t^{\prime}\right)
$$

and iterating once, we obtain an approximate solution of (4.8)

$$
\begin{aligned}
\boldsymbol{P}\left(t^{\prime}\right) & \approx-\int \mathrm{d} t^{\prime} \Gamma\left(t-t^{\prime}\right) \boldsymbol{E}\left(t^{\prime}\right) \\
& -\alpha \int \mathrm{d} t^{\prime} \Gamma\left(t-t^{\prime}\right)\left[\int \mathrm{d} t_{1} \Gamma\left(t^{\prime}-t_{1}\right) \boldsymbol{E}\left(t_{1}\right) \cdot \int \mathrm{d} t_{2} \Gamma\left(t^{\prime}-t_{2}\right) \boldsymbol{E}\left(t_{2}\right)\right] \\
& \times \int \mathrm{d} t_{3} \Gamma\left(t^{\prime}-t_{3}\right) \boldsymbol{E}\left(t_{3}\right) .
\end{aligned}
$$

It follows from (4.10) that the third-order susceptibility can be written as

$$
\begin{aligned}
& \chi^{(3)}\left(t-t_{1}, t-t_{2}, t-t_{3}\right) \\
& \quad=-\alpha \int \mathrm{d} t^{\prime} \Gamma\left(t-t^{\prime}\right) \Gamma\left(t^{\prime}-t_{1}\right) \Gamma\left(t^{\prime}-t_{2}\right) \Gamma\left(t^{\prime}-t_{3}\right),
\end{aligned}
$$

which as the function of frequencies [10] reads

$$
\begin{aligned}
\chi^{(3)}(\omega & \left.=\omega_{1}+\omega_{2}+\omega_{3}\right) \\
= & -\alpha \int \mathrm{d} t_{1} \mathrm{~d} t_{2} \mathrm{~d} t_{3} \mathrm{e}^{\mathrm{i} \omega_{1}\left(t-t_{1}\right)} \mathrm{e}^{\mathrm{i} \omega_{2}\left(t-t_{2}\right)} \mathrm{e}^{\mathrm{i} \omega_{3}\left(t-t_{3}\right)} \\
& \times \int \mathrm{d} t^{\prime} \Gamma\left(t-t^{\prime}\right) \Gamma\left(t^{\prime}-t_{1}\right) \Gamma\left(t^{\prime}-t_{2}\right) \Gamma\left(t^{\prime}-t_{3}\right)
\end{aligned}
$$


Substituting the Fourier representations of $\Gamma(t)(2.4)$ and performing the time integration, we obtain

$$
\chi^{(3)}\left(\omega=\omega_{1}+\omega_{2}+\omega_{3}\right)=-\alpha \widetilde{\Gamma}\left(\omega_{1}+\omega_{2}+\omega_{3}\right) \widetilde{\Gamma}\left(\omega_{1}\right) \tilde{\Gamma}\left(\omega_{2}\right) \tilde{\Gamma}\left(\omega_{3}\right) .
$$

More extensive description of the nonlinear effects in the framework of path integrals is now under investigation and will be published elsewhere.

\section{Conclusions}

We have shown here how an effective theory of the macroscopic electromagnetic field in a dispersive medium can be formulated with the help of the Feynman path integrals over classical histories of the system. As a natural starting point the microscopic Lagrangian of the Hopfield type [3], amended by coupling with the reservoir oscillators to account for losses [4], has been used. The effective Lagrangian, nonlocal in time, can be obtained in the explicit form in the case of a linear medium, when all functional integrations are of Gaussian type. As the main result at this level of calculation we have evaluated the frequency dependent dielectric function of the form which agrees with that obtained earlier by canonical quantization method [4]. Specific shape of $\varepsilon(\omega)$ as function of frequency depends on the form of the coupling with the reservoir fields. In particular, it has been shown here that the standard Lorentz model corresponds to the uniform, frequency independent coupling. Independent of the form of the coupling to the reservoir fields, the dielectric function has the Lorentz shape in a vicinity of the resonance frequency, according to formulae (3.5) and (3.6).

The functional integration methods are also very suitable and promising in the treatment of nonlinear effects in dispersive dielectric media, in particular those with a Kerr-type nonlinearity. Since the functional integrals are not Gaussian in this case and cannot be performed explicitly, it is necessary to use some sort of perturbative methods. We propose here to use an effective action for the polarization field $\boldsymbol{P}(t)$, obtained by integrating over the reservoir fields, when the polarization field is treated as a dynamical variable and the electric field is assumed to be an external field with given dependence on space and time variables. This effective action was further used to derive an integro-differential equation for $\boldsymbol{P}(t)$, with a first-order approximate solution corresponding to the linear case, and, when iterated once, giving the contribution cubic in the external electric field describing the Kerr-type nonlinearity. Having the third-order contribution to the polarization fields a standard method [10] was used to calculate the third-order susceptibility [cf. formula (4.13)]. Application of the functional integration method to the description of effective electric field in nonlinear media on macroscopic level requires further consideration and is presently being analyzed in more details. 


\section{References}

[1] R.P. Feynman, Rev. Mod. Phys. 20, 367 (1948).

[2] R.P. Feynman, A. Hibbs, Quantum Mechanics and Path Integrals, McGraw-Hill, New York 1965.

[3] J.J. Hopfield, Phys. Rev. 112, 1555 (1958).

[4] B. Huttner, S.M. Barnett, Phys. Rev. A 46, 4306 (1992).

[5] A. Bechler, J. Mod. Opt. 46, 901 (1999).

[6] S.M. Dutra, K. Furuya, Europhys. Lett. 43, 13 (1998); Phys. Rev. A 57, 3050 (1998).

[7] M. Wubbs, L.G. Suttorp, Phys. Rev. A 63, 043809 (2001).

[8] E. Schmidt, J. Jeffers, S.M. Barnett, L. Knoll, D.-G. Welsch, J. Mod. Opt. 45, 377 (1998).

[9] I. Białynicki-Birula, Z. Białynicka-Birula, Quantum Electrodynamics, Pergamon, Oxford 1975.

[10] Y.R. Shen, The Principles of Nonlinear Optics, Wiley, New Jork 1984. 